Editorial

\title{
Special Issue "Optical Fiber Interferometric Sensors: New Production Methodologies and Novel Applications"
}

\author{
M. Fátima Domingues ${ }^{1, *(\mathbb{D})}$, Nélia Alberto ${ }^{1}$ (i) and Paulo André ${ }^{2}$ \\ 1 Instituto de Telecomunicações and University of Aveiro, Campus Universitário de Santiago, \\ 3810-193 Aveiro, Portugal; nelia@ua.pt \\ 2 Department of Electrical and Computer Engineering and Instituto de Telecomunicações, \\ Instituto Superior Técnico, University of Lisbon, 1049-001 Lisbon, Portugal; paulo.andre@lx.it.pt \\ * Correspondence: fatima.domingues@ua.pt
}

check for

updates

Citation: Domingues, M.F.; Alberto, N.; André, P. Special Issue “Optical Fiber Interferometric Sensors: New Production Methodologies and Novel Applications". Photonics 2021, 8, 389. https://doi.org/10.3390/

photonics 8090389

Received: 30 August 2021

Accepted: 10 September 2021

Published: 14 September 2021

Publisher's Note: MDPI stays neutral with regard to jurisdictional claims in published maps and institutional affiliations.

Copyright: (C) 2021 by the authors. Licensee MDPI, Basel, Switzerland. This article is an open access article distributed under the terms and conditions of the Creative Commons Attribution (CC BY) license (https:// creativecommons.org/licenses/by/ $4.0 /)$.

\begin{abstract}
The collection of papers presented in this Special Issue (SI) portraits the state-of-the-art of photonic-based interferometric sensors, where new application areas were explored (such as spirometry) and novel sensitivity limits were achieved, using innovative sensing techniques for the monitoring of parameters, such as displacement, temperature or salinity.
\end{abstract}

Keywords: interferometric sensors; optical fiber; photonics

\section{Introduction}

Optical fiber interferometric sensors have been an expanding research topic in recent years, mainly due to its ability to perform measures with extreme accuracy on a variety of physical parameters, which range from temperature, strain, pressure, and chemical quantities to physiological parameters. For this Special Issue (SI), both original research papers and review articles describing the current state-of-the-art in this research field were requested and welcome, with the aim to provide a collection of high-quality papers, reporting on the present status and future outlook of the following topics:

- New and/or low-cost interferometers production methods;

- Novel optical fiber Fabry-Perot, Mach-Zehnder, Michelson, and Sagnac-based sensors;

- Optical fiber interferometric-based sensing for physical and chemical parameters;

- Optical fiber interferometric systems with microfluid integration;

- Low-cost, miniaturized, selective and multiparameter optical fiber interferometric devices;

- New bio/chemical probes for biomedical applications;

- Wearable/biomedical interferometric sensors;

- Advanced signal processing techniques;

- New interrogation techniques for interferometric sensors;

- New applications of optical fiber interferometric sensors.

The contributions to this SI resulted in a collection of 12 papers reporting on the advances in the research of interferometric optical fiber-based sensors and its novel applications. The scope of this SI intends to provide a synopsis of the current research trends and state-of-the-art in this field, covering recent technological improvements, new production methodologies and emerging applications, for researchers coming from different fields of science and industry.

The manuscripts published under this SI report on topics that range from interferometric sensors for thickness and dynamic displacement measurement, up to pulse wave (PW) and spirometry applications. A brief overview of the collection of papers present in this SI will be described in the following section.

We appreciate the authors' contributions to this SI, which gathers original papers with a comprehensive and up-to-date overview on a variety of topics, with the common denominator based on new optical fiber and photonic-based interferometric sensors. 
Additionally, we would like to thank the academic editors and reviewers for their efforts and contributions to guarantee the scientific rigor and quality of the manuscripts published and, consequently, of this SI.

\section{Contributed Papers}

The papers collected in this SI reflect the importance of the topic for the research community, and the wide field of applications in which such novel technological advances can have improved performance.

Among the diverse fields of applications gathered in this SI, Gao et al. reported on a speckle interferometry system for micro-electromechanical systems (MEMs) dynamic displacement measurement. They proposed a large-field microscopic speckle interferometer, based on two embedded doublet lens groups for the realization of an improved optical Mach-Zehnder system. With the proposed solution, the authors can expand the field of view, while reducing the coherent noise of the reflected signal, achieving a high precision and real time dynamic measurement of MEMS [1].

On a different application, the authors from [2] reported on the use of digital grating Moiré effect, to be applied in the measurement of the curvature radius and focal lengths of biconvex and biconcave spherical lenses and mirrors. With the proposed methodology, they proved to be able to provide measurements of focal length and a radius of curvature with errors below $0.5 \%$ [2].

The authors of [3] report on the development and implementation of an optical contactless technique to monitor the production of plastic films which thicken in real time. The developed apparatus is a low-coherence fiber interferometer, based on a cross between an auto-correlator and a white light interferometer, which is able to measure blown film thickness during production [3].

Stolarik et al. contributed to this SI with a report on promising alternatives for vibration measurements, with an optical fiber Michelson interferometer, designed with passive demodulation technique using three mutually phase-shifted optical outputs [4]. The performance of this device was compared with existing seismic devices, and the results demonstrate its great potential, with correlation coefficients above 0.9 , and average deviations of the amplitude of the oscillation velocity around 0.02. [4].

Another paper that contributed to the success of this SI, from the authors of [5], reports on different optical fiber-based interferometric techniques to monitor the PW signal. The authors provide an exhaustive comparison of performance (signal to noise ratio, repeatability, and suitability of demodulated signals), of the optical fiber sensors based on fiber Bragg grating (FBG), Fabry-Perot interferometer (FPI), single mode-multimode-single mode intermodal interferometer and optical coherence tomography (OCT), to monitor the PW signal [5]. From the presented study, it was concluded that OCT-based sensors outperform the other solutions in terms of signal demodulation quality and robustness and presented a clear discussion of the advantages and drawbacks of all compared PW measurement approaches [5].

Marques et al., contributed to this SI, with a manuscript in which the suitability of the Master-Slave (MS) procedures for processing/calibration of the signal from interferometers, driven by akinetic sources, that exhibit non-linear tuning, was evaluated [6]. The authors explore the use of two different MS-processing techniques for the akinetic source calibration, being able to recover, in both cases, the spectral depth information, enabling an improved analysis of phase errors, which may occur around invalid-to-valid transactions or due to the source performance [6].

Additionally, an enhanced temperature sensor, based on a dual-core fiber with an eccentric core and a central core in-fiber Mach-Zehnder sensor, was reported by Qiu et al. [7]. The central core effective refractive index is modulated by the glycerol-water solution in the fluidic channel, adjacent to the central core. The sensor-transmitted spectra are shifted, according to the temperature variations, with a sensitivity of $2.77 \mathrm{~nm} /{ }^{\circ} \mathrm{C}$ (for a channel with $15 \mathrm{~cm}$ ). The authors also found a relation between the sensitivity of the sensor and the 
length of the fluidic channel, showing that the longer the channel, the higher the sensor's sensitivity (a length of $29.5 \mathrm{~cm}$ will provide an increased sensitivity of $5.69 \mathrm{~nm} /{ }^{\circ} \mathrm{C}$ ) [7].

The report on a high sensitivity displacement interferometer-based sensor, combining the Vernier effect and the push and pull method, was reported in [8]. The combination of these two methodologies, and the optimization of the two interferometers, resulted in a sensitivity of $254 \pm 6 \mathrm{~nm} / \mu \mathrm{m}$, a magnification factor (M) of 1071 and an $M_{\text {Vernier }}$ of 1.9 [8].

Additionally, the development of solutions to monitor seawater salinity was presented by Zhou et al. The solution proposed is based on a novel microfiber coupler interferometer, produced by connecting the two out ports of a microfiber coupler, to Faraday rotating mirrors. The proposed solution exhibited a spectral red shift with a water salinity increase, with the highest sensitivity reaching $303.7 \mathrm{pm} / \%$ and a resolution of $0.03 \%$ [ [9].

Another temperature sensor, based on a photonic crystal fiber (PCF) Sagnac interferometer, was presented in [10], where the authors, theoretically and experimentally, reported on a liquid-filled highly asymmetric PCF structure for temperature monitoring. The proposed structure presented a sensitivity of $17.53 \mathrm{~nm} /{ }^{\circ} \mathrm{C}$ with a resolution of $5.7 \times 10^{-4}{ }^{\circ} \mathrm{C}[10]$.

As part of this SI collection, is also the review manuscript provided by Hillmer et al., focusing on a detailed comparison (spectral range required, resolution, tunability, efficiency, multiplexing capabilities, miniaturization limits, and potential of nanoimprint), of different miniaturized sensor concepts for nanoimprint. The studyranges from classical optical transmission and reflection gratings, up to fiber tip sensors, also exploring static FPI filter arrays, plasmonic filters and MEMS tunable FPI interferometers. The work presented by these authors provides a thorough analysis of the above-mentioned technologies, and its potential to be fabricated by nanoimprint techniques [11].

Finally, a novel application of FPI-based sensors was reported by Nepomuceno et al., where the authors presented an optical fiber FPI-based, 3D printed device to evaluate the individual pulmonary capacity by spirometry [12]. The spirometer is based on the conversion of the air flow into strain variations applied to the optical fiber, which modulates the FPI spectral response. In this contribution, an exhaustive theoretical analysis of the device, as well as its preliminary results, is presented, from which it was possible to acquire spirometry critical parameters: forced expiratory volume in $1 \mathrm{~s}$ (FEV1), forced vital capacity (FVC), FEV1/FVC index and the peak expiratory flow (PEF) [12].

\section{Outlook and Prospects}

The papers collected in this SI represent only a small window of the current interest and developments in photonic-based sensors, with a particular focus on interferometric devices. The development of new production methodologies, targeting more cost effective and less complex production lines, still has room to grow. Innovative and promising developments of the production methods, devices and techniques for spectral analysis, and new areas of application are to be expected for this type of sensing device.

Author Contributions: M.F.D. wrote the manuscript draft. N.A. and P.A. contribute to the manuscript revision. All authors have read and agreed to the published version of the manuscript.

Funding: This work is funded by FCT/MCTES through national funds and when applicable cofounded by EU funds under the UIDB/50008/2020-UIDP/50008/2020. This work is also funded by FCT/MEC through national funds and when applicable co-funded by the FEDER-PT2020 partnership agreement under the project UID/EEA/50008/2019.

Acknowledgments: M. Fátima Domingues and Nélia Alberto acknowledge the REAct (FCT-IT-LA) and PREDICT (FCT-IT-LA) scientific actions, respectively.

Conflicts of Interest: The authors declare no conflict of interest. 


\section{References}

1. Gao, C.; Gao, Z.; Niu, Y.; Wang, X.; Zhao, J.; Deng, L. An Improved Large-Field Microscopic Speckle Interferometry System for Dynamic Displacement Measurement of MEMS. Photonics 2021, 8, 271. [CrossRef]

2. Han, C.-Y.; Lo, W.-T.; Chen, K.-H.; Lee, J.-Y.; Yeh, C.-H.; Chen, J.-H. Measurement of Focal Length and Radius of Curvature for Spherical Lenses and Mirrors by Using Digital-Grating Moiré Effect. Photonics 2021, 8, 252. [CrossRef]

3. Norgia, M.; Pesatori, A. Interferometric Instrument for Thickness Measurement on Blown Films. Photonics 2021, 8, 245. [CrossRef]

4. Stolarik, M.; Nedoma, J.; Martinek, R.; Kepak, S.; Hrubesova, E.; Pinka, M.; Kolarik, J. New Methods to Seismic Monitoring: Laboratory Comparative Study of Michelson Fiber-Optic Interferometer and Pneumatic Measurement Systems. Photonics 2021, 8, 147. [CrossRef]

5. Ushakov, N.; Markvart, A.; Kulik, D.; Liokumovich, L. Comparison of Pulse Wave Signal Monitoring Techniques with Different Fiber-Optic Interferometric Sensing Elements. Photonics 2021, 8, 142. [CrossRef]

6. Marques, M.J.; Cernat, R.; Ensher, J.; Bradu, A.; Podoleanu, A. Akinetic Swept-Source Master-Slave-Enhanced Optical Coherence Tomography. Photonics 2021, 8, 141. [CrossRef]

7. Qiu, H.; Zhao, C.; Hu, X.; Chen, H.; Yu, Q.; Lian, Z.; Qu, H. Glycerol-Water Solution-Assisted Mach-Zehnder Temperature Sensor in Specialty Fiber with Two Cores and One Channel. Photonics 2021, 8, 103. [CrossRef]

8. Robalinho, P.; Frazão, O. Giant Displacement Sensitivity Using Push-Pull Method in Interferometry. Photonics 2021, 8, 23. [CrossRef]

9. Zhou, L.; Yu, Y.; Huang, H.; Tao, Y.; Wen, K.; Li, G.; Yang, J.; Zhang, Z. Salinity Sensing Characteristics Based on Optical Microfiber Coupler Interferometer. Photonics 2020, 7, 77. [CrossRef]

10. Monfared, Y.E.; Ahmadian, A.; Dhasarathan, V.; Liang, C. Liquid-Filled Highly Asymmetric Photonic Crystal Fiber Sagnac Interferometer Temperature Sensor. Photonics 2020, 7, 33. [CrossRef]

11. Hillmer, H.; Woidt, C.; Kobylinskiy, A.; Kraus, M.; Istock, A.; Iskhandar, M.S.Q.; Brunner, R.; Kusserow, T. Miniaturized Interferometric Sensors with Spectral Tunability for Optical Fiber Technology-A Comparison of Size Requirements, Performance, and New Concepts. Photonics 2021, 8, 332. [CrossRef]

12. Nepomuceno, A.C.; Paixão, T.; Alberto, N.; André, P.S.d.B.; Antunes, P.; Domingues, M.F. Optical Fiber Fabry-Perot Interferometer Based Spirometer: Design and Performance Evaluation. Photonics 2021, 8, 336. [CrossRef] 